\title{
Transcriptome Analysis Revealed the Regulation of Gibberellin and the Establishment of Photosynthetic System Promote Rapid Seed Germination and Early Growth of Seedling in Pearl Millet
}

\section{Bingchao Wu}

Sichuan Agricultural University

Min Sun

Sichuan agricultural university

Huan Zhang

Sichuan Agricultural University

Dan Yang

Sichuan Agricultural University

Chuang Lin

Sichuan Agricultural University

Imran Khan

Sichuan Agricultural University

\section{Xiaoshan Wang}

Sichuan Agricultural University

Xinquan Zhang

Sichuan Agricultural University

\section{Gang Nie}

Sichuan Agricultural University

\section{Guangyan Feng}

Sichuan Agricultural University

\section{Yanhong Yan}

Sichuan Agricultural University

\section{Zhou Li}

Sichuan Agricultural University

\section{Yan Peng}

Sichuan Agricultural University

Linkai Huang ( $\square$ huanglinkai@sicau.edu.cn )

Sichuan Agricultural University https://orcid.org/0000-0001-6622-5841 
Research

Keywords: Pearl millet, Seed germination, Seedling growth, Transcriptome, Hormone signal transduction

Posted Date: December 11th, 2020

DOI: https://doi.org/10.21203/rs.3.rs-125050/v1

License: (c) (i) This work is licensed under a Creative Commons Attribution 4.0 International License. Read Full License

Version of Record: A version of this preprint was published at Biotechnology for Biofuels on April 11th, 2021. See the published version at https://doi.org/10.1186/s13068-021-01946-6. 


\section{Abstract}

Background冈Seed germination is the most important stage for the formation of a new plant. This process starts when the dry seeds begin to absorb water and ends when the radicles sticks out. The germination rate of different plant seeds varies differently. Most energy plants that usually grow on marginal land, the rapid germination of seeds is more conducive to its superiority in competition with surrounding plants, which is also the guarantee of normal plant development and high yield. Pearl millet is an important cereal crop that shares widespread applications in the world. It has the advantages of fast growth, high yield, and low maintenance cost. It can also be used to extract bioethanol to solve the increasingly prominent energy problems. Previous germination experiments are the evidence of very fast seed germination rate of pearl millet, but the molecular mechanisms behind it is still unclear.

Results: Through the germination test and the measurement of the germs and radicles length of the seedlings, we found that pearl millet seeds germinated very quickly after 24 hours of swelling of the dry seeds. By using transcriptome sequencing technology, we characterized the gene expression patterns of dry seeds, water imbibed seeds, germs and radicles of seedlings, and found the more DEGs in radicles than germs. Further analysis showed that different genome clusters function specifically at different tissues and time periods. WGCNA and KEGG enrichment analysis showed that that many genes that positively regulate plant growth and development are highly enriched and expressed, especially the gibberellin signaling pathway that can promote seed germination. We speculated that the activation of these key genes promotes the germination of pearl millet seeds and the growth of seedlings. To verify this inference, we measured the content of the main effect hormone gibberellin and found that the gibberellin content after seed imbibition rose sharply and remained at a high level.

Conclusions $\triangle T$ This study explored the expression patterns of genes involved in pearl millet growth from the germination of dry seeds to the early growth stages. Also identifies the key genes involved in the regulation of seed germination and seedling growth. The activation of key genes in these pathways may contribute to the rapid germination and growth of seeds and seedlings in pearl millet. These results provide new insights to solve the problem for the plants with slow seed germination and seedling growth.

\section{Background}

Seed germination represents the emergence of new plants. This is a complicated physiological process that is affected by both the internal development of the seeds and changes in the external environment [1]. The germination begins with the absorption of water by seeds and ends with the initiation of hypocotyl elongation [1]. After the seed germination, growth begins with the appearance of radicles and this growth refers to seedling growth [2]. According to the definition, the rigorous germination process includes the beginning of the dry seed's absorption of water to the extension of the radicles and the termination of the elongation of the hypocotyl [1]. In order to avoid competing for land with crops, energy plants are usually need to plant on marginal land, where resources are very limited. Plants in this environment tend to compete for sunlight, air, water and so on. Therefore, the rapid germination of seeds 
is very important, which can improve the ability of plants to compete with other weeds in the field. Moreover, the rapid germination of seeds involves many physiological and biochemical reactions, which are often controlled by genes. It is necessary to understand the reasons for the rapid seed germination that will help us to improve the scale of plant cultivation and economic benefits, and provide a reference for solving problems for plants with slow seed germination.

Fossil fuels have been using for a long time to fulfil the demand of energy and they still dominate energy acquisition, but their negative effects cannot be ignored. It is an obvious risk to environmental governance and energy security. Therefore, a big part of world population has shifted their attention from fossil fuels to bioenergy [3]. Pearl millet (Pennisetum glaucum (L.) R. Br.), an important cereal/bioenergy crop contributes to variety of application in the world. It is widely planted all over the world with fast growth, high yield and strong resistance to various environmental stresses. Pearl millet is rich in easy-toextract fermented sugars, which can be used to produce bioethanol, thus, many researchers regard it as a very potential energy plant [4-6]. It is also one of the parents of the important energy plant hybridized pennisetum. At the same time, pearl millet is also the sixth largest cereal crop in the world [7]. Through our germination experiments, we found that the seed germination and seedling growth rate of pearl millet is extremely fast, which is significantly faster than maize (Zea mays L.), rice (Oryza sativa L.), orchardgrass (Dactylis glomerata L.), switchgrass (Panicum virgatum L.) and so on (Figure S1 and Table S1). However, the pearl millet is considered as the crop with fast seed germination rate and the reason behind its fast germination rate is still unknown.

Plant hormones including gibberellin acid (GA), auxin, and cytokinin (CTK) are organic signaling molecules that are produced by plants through their own metabolism and they actively participate in various physiological processes. They can function at the place of synthesis and can also be transported to distant parts of the plant[8]. Plant hormones can independently or coordinate with each other to regulate the growth and development of plants [9]. Many studies found that abnormalities in their synthesis pathways or signal transduction pathways will cause defects in plant growth and development [10]. Among these hormones, abscisic acid (ABA) and GA are the dominant factors for seed dormancy and germination, respectively. ABA promotes seed dormancy, while GA helps the seeds to break dormancy and promote germination $[11,12]$. For example, some studies have reported the lower content of GA in the seeds of mutant ga 1 whose GA synthesis was blocked due to which the germination was incomplete even under normal conditions [13]. Another study revealed that fast-growing seedlings of switchgrass accumulated higher GA content than slow-growing seedlings. [14]. Auxin mainly regulates the development of plant leaves and roots $[15,16]$. Some studies have found that auxin can maintain root stem cells by inducing the accumulation of transcription factor PLT1/2 [17]. In addition, the formation of adventitious roots is also regulated by auxin in rice [18]. Cytokinin is also involved in the development of plant roots. Overexpression of cytokinin A response factors can promote the growth of Arabidopsis roots [19]. The similar result was also reported in rice [20]. Some pieces of evidence showed that the growth of adventitious roots in rice significantly decreases after the treatment with cytokinin synthesis inhibitors, indicating that cytokinin behaves like auxin and involves in regulating the 
development of adventitious roots. [21]. However, whether these hormones regulate pearl millet seed germination and seedling growth through signal transduction pathways is still unknown.

The growth of plants is inseparable from photosynthesis, and the factors that affect photosynthesis may also influence the growth and development of plants [22]. Plants synthesize the organic matter through photosynthesis for their own growth and development. Previously, it has been described that increasing the daily light integral (DLI) within a certain range can improve the growth of plants, promote the accumulation of nutrients, and accelerate the development process [23, 24]. As an important part of the photosynthetic system, photosynthetic antenna proteins contain different pigments composition and has absorption characteristics for different lights, thus they increase the efficiency of photosynthesis [25]. The early growth rate of pearl millet seedlings is very rapid, so we can speculate that this is closely related to the sufficient energy supply of pearl millet seedlings. However, it is still unknown whether the photosynthesis system has been formed and activated at this stage or not.

Transcriptome sequencing technology has become an effective means to understand the relationship between gene expression patterns and phenotypes [26-29]. The transcriptome sequencing at different time points, tissues of germinated seeds and seedling growth in pearl millet will help us to identify the key genes involved at this stage. For example, a study on the transcriptome dynamics of leaves during the germination of corn seeds found that gene clusters involved in hormonal metabolism and signal transduction have different expressions at different time points [30]. In this study, we conducted a seed germination experiment and found that pearl millet seeds reached the germination standard at $24 \mathrm{~h}$ or even earlier than $24 \mathrm{~h}$ after imbibition, and the radicles elongation speed was faster than that of the germs. Based on transcriptome data, it is found that number of DEGs in the radicles are more than that in the germs. Further WGCNA analysis was performed on all DEGs which revealed, that different gene clusters were time and tissue specific. Finally, by comparing with previous research results, we found that the high expression of key genes involved in hormone signal transduction, photosynthesis, photosynthesis-antenna proteins and brassinosteroid synthesis pathway may be the reasons for the rapid germination and growth of seeds and seedlings in pearl millet. The sharp increase of GA content after seed imbibition confirmed the activation of the gibberellin signal transduction pathway and resulted in the rapid germination of seed. In summary, this study identifies key genes involved in the seed germination and early stages of seedling growth in pearl millet. These results can provide a reference for solving problems for plants with slow seed germination and seedling growth.

\section{Results}

\section{The rate of seeds germination and seedlings growth is extremely fast in pearl millet}

In the studies of seed germination, we found that pearl millet seedlings grow very fast under suitable temperature and light conditions (Fig. 1, Figure S1 and Table S1). We speculated that a series of rapid physiological and biochemical reactions occurred during the very early stage of seedlings development 
due to which its growth increases. In order to study the reasons for the fast germination rate, we focused our attention on 5 time points in the early germination period: 1. the dry seed. 2. 2 hours after imbibition (still in seed form). 3-5. 24, 36 and 48 hours after imbibition (radicles and germs appeared). We measured the length of germs and radicles of seedlings at 24,36 and 48 hours after imbibition to characterize the growth rate of seedlings.

The length of germ reaches one-half of the seed length and length of radicle reaches the seed length is regarded as germination. The results showed that the rate of germination in pearl millet seeds is very quick (Fig. 1, Table S2). At $24 \mathrm{~h}$ or even earlier than $24 \mathrm{~h}$ after seed imbibition, the length of germs and radicles have reached the germination standard, and growth rate of radicles was faster than that of germs, especially during the period from $24 \mathrm{~h}$ to $36 \mathrm{~h}$ (Fig. 1a). During the period of $24 \mathrm{~h}$ to $48 \mathrm{~h}$, the seedlings experienced a very rapid growth process, and there were significant differences in the germ length and root length between among time points (Fig. 1b). These results suggested that the pearl millet seeds have gained some advantages after imbibition, which endows the seeds with ability of rapid germination and seedling growth. This indicates that we can study the transcriptome differences during this period in the next step, which will help to better understand the mechanism of pearl millet seed germination and seedling growth.

\section{More DEGs in the radicles than in the germ}

Through transcriptome sequencing, a total of 810,182,656 raw data were generated from 24 samples, ranging from 34402297 to 42543864 . We uploaded the original data to NCBI database(PRJNA670183). After filtering the original data, we got $779,909,975$ clean data, ranging from 33395607 to 41346841 . The GC content of all samples ranges from $52.57-57.59 \%$, Q20 ranges from $96.93-98.04 \%$, and Q30 ranges from $92.01-94.66 \%$ (Table S3), indicating the high sequencing quality and the obtained data can be used for subsequent analysis.

We used the full-length transcriptome sequence of pearl millet as a reference sequence for alignment and gene expression calculation [6]. A great consistency between different biological replicates of each sample is a prerequisite to ensure the reliability of subsequent bioinformatics analysis results. Therefore, we calculated Pearson's correlation coefficient for different samples and found that the three biological replicates between each sample have good correlations, and the correlation coefficients are all greater than 0.9 ( $P$ value $<0.01)$ (Figure S2, Table S4). In order to understand the dynamics of gene expression changes in seed, germ and radicles at different time points, we divided the 16 comparison groups into four categories for differentially expressed genes analysis (Table 1). A total of 29,514 DEGs were identified in the germ, of which only 58 genes were shared among all time points, while a total of 30,263 DEGs were identified in the radicles, of which only 30 genes were shared among all time points (Fig. 2). It is worth noting that in both the radicles and the germ, in the $24 \mathrm{HAl}$ and $2 \mathrm{HAl}$ comparison groups (24HAIG:2HAIS and 24HAIR:2HAIS), there are more down-regulated genes than up-regulated genes, and the total number of DEGs were also the largest, while all other comparison groups have more up- 
regulated genes than down-regulated genes. This may indicate that the internal gene expression is very strong after the seed imbibition for 2 hours. We also found that, except for the 24HAl: $2 \mathrm{HAl}$ and the 48HAl: $36 \mathrm{HAl}$ comparison group after seed imbibition, the number of DEGs in the radicles was greater than that in the germ in other comparison groups. This indicated that the gene expression in the radicles was more active, which may explain why the radicles appear earlier than the germ, and the faster growth rate than germ. Since it was found in the morphological measurement that the elongation of the radicles was significantly greater than that of the germ at $36 \mathrm{~h}$ compared with $24 \mathrm{~h}$, we checked the DEGs of the germ and the radicles in $36 \mathrm{HAl}: 24 \mathrm{HAl}$ groups, and found that the number of DEGs in the radicles were 6644 , which was higher than 3447 in the germ. Then we compared the germs and radicles at 3 time points and found that the number of DEGs between radicles and germ were 12697 in 36HAIG:36HAIR group, which was significantly higher than the 7239 and 8546 of $24 \mathrm{HAl}$ and $48 \mathrm{HAl}$. This may explain why the elongation of the radicles is much greater than that of the germ at $36 \mathrm{HAl}$.

Table 1

Summary of DEGs among 16 comparison groups

\begin{tabular}{|lllll|}
\hline Sort & $\begin{array}{l}\text { comparison } \\
\text { groups }\end{array}$ & $\begin{array}{l}\text { Number of up- } \\
\text { regulated genes }\end{array}$ & $\begin{array}{l}\text { Number of down- } \\
\text { regulated genes }\end{array}$ & $\begin{array}{l}\text { Total number } \\
\text { of DEGs }\end{array}$ \\
\hline Seeds & 2HAIS:0 & 10595 & 5825 & 16420 \\
\hline Germs & 24HAIG:0 & 8807 & 6468 & 15275 \\
\hline 36HAIG:0 & 10026 & 7082 & 17108 \\
\hline 48HAIG:0 & 11049 & 5879 & 16928 \\
\hline 24HAIG:2HAIS & 10043 & 11917 & 21960 \\
\hline 36HAIG:24HAIG & 2457 & 990 & 3447 \\
\hline 48HAIG:36HAIG & 805 & 513 & 1318 \\
\hline 24HAIR:0 & 9828 & 7324 & 17152 \\
\hline 36HAIR:0 & 12646 & 6458 & 19104 \\
\hline 48HAIR:0 & 12853 & 6806 & 19659 \\
\hline 24HAIR:2HAIS & 9703 & 11404 & 21107 \\
\hline 36HAIR:24HAIR & 4526 & 2118 & 6644 \\
\hline 48HAIR:36HAIR & 386 & 142 & 528 \\
\hline 24HAIG:24HAIR & 3699 & 3540 & 7239 \\
\hline 36HAIG:36HAIR & 5538 & 7159 & 12697 \\
\hline 48HAIG:48HAIR & 4938 & 3608 & 8546 \\
\hline
\end{tabular}




\section{The regulation mechanism of seed germination is specific at different stages}

In order to better identify the DEGs related to different time points and their expression trends in the radicles and germ, the standardized expression data of 24307 genes were analyzed by gene coexpression network from 24 samples (three biological replicates). A total of 19 modules were generated, and each color represents a module,which is a cluster of highly related genes (Fig. $3 a$ and $3 b$ ). We found 4 modules with specific time or tissue expression, namely "midnightblue", "cyan", "turquoise" and "brown" (Fig. 3c). The brown module contains 1409 genes which are mainly expressed in dry seeds; the turquoise module contains 5577 genes which are mainly expressed at 2HAIS; the midnightblue module contains 902 genes which are mainly expressed in germ at 24,36 and $48 \mathrm{HAl}$; the cyan module contains 4498 genes which are mainly expressed in the radicles at 36 and $48 \mathrm{HAl}$.

In order to further understand the biological functions of these 4 modules, we performed KEGG analysis on the genes in these 4 modules (Fig. 3d). The genes in the brown module are significantly enriched in alanine, aspartate and glutamate metabolism (ko00250), Pyrimidine metabolism (ko00240), Valine, leucine and isoleucine degradation (ko00280) and RNA polymerase (ko03020), etc. The genes in the turquois module are significantly enriched in 13 pathways including Plant hormone signal transduction (ko04075) and MAPK signaling pathway-plant (ko04016). The genes in the Midnightblue module are enriched in 11 pathways such as Photosynthesis-antenna proteins (ko00196), Porphyrin and chlorophyll metabolism (ko00860), Photosynthesis (ko00195), Carbon metabolism (ko01200) and Carbon fixation in photosynthetic organisms (ko00710). The genes in the Cyan module are significantly enriched in 17 pathways including Phenylpropanoid biosynthesis (ko00940), Flavonoid biosynthesis (ko00941) and Brassinosteroid biosynthesis (ko00905) (Fig. 3d, Table S4). It is worth noting that the pathways shared by genes in the four modules are very few (Figure S3, Table S5). The turquoise and cyan modules shared two pathways, namely taurine and hypotaurine metabolism (k00430) and tryptophan metabolism (ko00380). Similarly, midnightblue and cyan modules also shared two pathways, namely biosynthesis of secondary metabolites (ko01110) and metabolic pathways (k001100), while the brown module didn't share pathways with any module. The above results indicated that the function of genes in the module has strong tissue and time specificity, and different genes are functioning at different stages. This indicated that exploring the regulation mechanism of each stage can help us better understand the reasons for rapid germination in pearl millet seeds.

\section{Function analysis of candidate genes related to seeds germination and seedling growth in pearl millet}

\section{The signal transduction of gibberellin, auxin and cytokinin promotes the rapid germination of pearl millet seeds}


Phytohormones are organic signaling molecules that are produced by plants through their own metabolism and induce obvious changes even at very low concentrations, and play a very important role in the regulation of plant growth and development. Plant hormones often activate the downstream gene expression through signal transduction pathways to regulate plant growth and development. Therefore, it is necessary to analyze the factors in these hormone signal transduction pathways.

GA is a type of diterpene compound, which plays an important role in the growth and development of plants, and has been proven by a large number of studies to regulate the process of seed germination. When GA works through the signal transduction pathway, the receptor GID1 first senses gibberellin, and then combines with DELLA protein to form a GID1/GA/DELLA complex, which relieves the inhibition of DELLA on key downstream regulatory factors such as PIF3 and PIF4, and then regulates various biological processes [31, 32]. Our results found that the expression of the two GID1 genes (i1_LQ_LWC_c23529/f1p0/1967 and i2_LQ_LWC_c18562/f1p4/2746) reached the highest at $2 \mathrm{~h}$ after seeds imbibition, and the same expression trend was also observed in the two PIF3 genes (i2_LQ_LWC_c21636/f1p4 /2470 and i2_LQ_LWC_c36807/f1p4/2205) and 3 PIF4 genes (i0_LQ_LWC_c2048/f1p68/923, i1_LQ_LWC_c35538/f1p6/1792 and i2_LQ_LWC_c83983/f1p0/2153) (Fig. $4 \mathrm{~b}$ ). Considering GA as the main hormone for seed germination, and the signal transduction pathway of this hormone is so highly active, we determined the GA content in the early stage after seed imbibition. We found that the content of GA in dry seeds was the lowest, began to rise after imbibition, and arrived the highest at 4HAIS. The difference between every two time points was extremely significant $(P<0.01)$ (Fig. 5, Table S6). This result showed that a large amount of GA was synthesized immediately after seed imbibition, and the downstream signal transduction pathway was also stimulated to promote seed germination rapidly.

Furthermore, auxin is the earliest discovered member of the plant hormones family. It is a general term for a class of compounds that include indole acetic acid (IAA) and have similar physiological effects to indole acetic acid. It is involved in many biological and physiological processes including growth and development, such as root and leaf $[15,17,33,34]$. When auxin works through the signal transduction pathway, auxin receptor TIR1 first senses auxin, then mediates the degradation of Aux/IAA protein, and then mediates the expression of downstream genes by regulating ARFs transcription factors to promote cell growth and regulate plant growth and development. In the current study, we found that the TIR1 gene (i5_LQ_LWC_c8187/f1p3/5450) and two ARF genes (i2_LQ_LWC_c82062/f1p4/3002 and i2_LQ_LWC_c126242/f1p4/2422) were highly expressed at 2HAIS. In addition, some studies have shown that the downstream gene $\mathrm{GH} 3$ of this pathway was up-regulated by the action of auxin, but it involved the degradation of auxin, so it showed a negative feedback effect on auxin. It is generally believed that GH3 gene plays an important role in auxin homeostasis [35]. The SAUR gene is thought to be involved in auxin-regulated cell expansion, and it has also been found to be highly expressed in the elongated hypocotyl [36, 37]. In our results, we also found that two GH3 genes (i2_HQ_LWC_c98146/f9p2/2256 and i2_LQ_LWC_c131239/f1p1/2110) and two SAUR genes (i1_LQ_LWC_c13114/f1p10/1086 and i1_LQ_LWC_c13186/f1p28/1222) have similar expression patterns (Fig. 4a). 
CTK has been involved in the regulation of plant cell division, growth and development of tissues, organs and individuals. The signal transduction pathway of cytokinin is firstly sensed by the histidine receptor kinase CRE1 (AHK2_3_4) to autophosphorylate histidine, and then the phosphate group is transferred to the aspartic acid residue in the self-receptive region. Then the phosphate group on the aspartic acid residue of the receptor is transferred to the histidine residue of the cytoplasmic histidine phosphorylation transfer protein (AHP). Finally, the phosphorylated histidine transfer protein enters the nucleus and transfers phosphate groups to A or B response factors (ARRs), among which B response factors (ARR-B) have transcription factor activity and can initiate downstream gene expression after phosphorylation [38-40]. Our research found that the expression levels of three CRE1 genes

(i4_HQ_LWC_c31467/f3p1/4211, i4_LQ_LWC_c5323/f1p3/4657 and i4_LQ_LWC_c16458/f1p0/4224) and three ARR-B genes (i2_HQ_LWC_c64881/f4p3/2784, i2_HQ_LWC_c72282/f2p2/2653 and i3_HQ_LWC_c29456/f2p0/3081) reached their peak at 2HAIS (Fig. 4c).

In summary, we found that in the signal transduction pathways of gibberellin, auxin, and cytokinin, the expression level of genes that play a positive role reached the highest level at 2HAIS in the pearl millet. Therefore, we speculated that after the seeds absorb water for a short time, the hormone signal transduction in the seeds became active, which promoted the germination of pearl millet seeds.

\section{The formation of photosynthetic system promotes the rapid growth of pearl millet seedlings}

Photosynthesis plays a very important role in the growth and development of plants. It converts solar energy into chemical energy and inorganic matter into organic matter and provides energy to plants needed for growth. It includes two light system. Photosystem I mostly produces the negative oxidationreduction reaction in nature, and to a large extent determines the amount of global enthalpy in the living system. Photosystem II produces an oxidant whose redox potential is sufficient to oxidize H2O, which is a very abundant substrate that can ensure an almost unlimited source of electrons for life on earth [41]. Both systems are multi-subunit supramolecular complexes, including a core complex and a peripheral antenna system [41, 42]. In plants, the peripheral antennas are all made up of the light-harvesting complex (LHC). LHCls (Lhca) and PSI core form a PSI-LHCl complex, and LHClls (Lhcb) and PSIl core form a PSII-LHCII complex. The antenna system has different pigment composition, so it has different light absorption characteristics [25]. The light-harvesting complex (LHCII) in PSII is the most abundant membranous protein on earth. It participates in the first step of photosynthesis, absorbs and transmits solar energy for photosynthesis on the chloroplast membrane, and plays a role in regulating photosynthesis and photoprotection $[43,44]$. Our results found that in the photosynthesis pathway, a total of 23 genes were identified to exist in different photosystems, and their expression levels reached the highest at 36HAIG (Fig. 6a). In the photosynthesis-antenna protein pathway, in addition to LHCA5, the other four light-harvesting complexes in LHCls (LHCA1,i0_LQ_LWC_C2012/f1p117/375; LHCA2,

i1_LQ_LWC_C27204/f1p0/1130; LHCA3, i1_HQ_LWC_c39810/f24p5/1138; LHCA4,

i1_LQ_LWC_c34601/f1p0/1459)were significantly enriched and expressed the highest at $36 \mathrm{~h}$ after seeds

Page $10 / 26$ 
imbibition in the germ. LHCB1 (i1_LQ_LWC_c19196/f1p0/1725, i1_LQ_LWC_c40686/f1p0/1092 and i1_LQ_LWC_c42565/f1p0/1078), LHCB4 (i1_HQ_LWC_c36891/f14p0/1217) and LHCB5

(i1_LQ_LWC_c26257/f1p0/1255) in LHC囚s also showed the same expression pattern (Fig. 6b). These results showed that the genes involved in photosynthesis and antenna protein genes in the germs have been highly expressed at 36 hours after the seeds imbibition. They generated a lot of energy for plant growth and utilization, and the antenna protein system promoted the transmission of solar energy and improved photosynthesis effectiveness of action. Therefore, we speculated that the rapid formation of the photosynthesis system helped the pearl millet to more effectively produce energy for growth and development, which is an important reason for the rapid growth of the seedlings in pearl millet.

\section{Brassinosteroids promote radicles elongation}

Brassinosteroid is a kind of widely distributed plant hormone, which plays an important role in almost all the growth process of plants, regulating the elongation and division of cells [45]. When rice lacks $B R$, its growth and development was affected, showing the stunted growth of plant [46]. The BR-insensitive mutants of Arabidopsis thaliana showed many defects during growth and development, including short plants and reduced apical dominance [45]. It was also found in Arabidopsis roots that the interaction of BR and auxin mediated by BRX (BREVIS RADIX), necessary for optimal root growth. The phenotype of the brx mutant is caused by the lack of root-specific BR. This defect affects about $15 \%$ of the root gene expression levels of all Arabidopsis genes, but the expression levels of these genes can be restored by BR treatment [47]. This suggested that the normal level of BR is essential for the development of plants, especially roots. In our research, we found that the key enzymes for biosynthesis with BR include CPD (i2_HQ_LWC_c41220/f2p0/2056 and i2_LQ_LWC_c11071/f1p0/2085), DET

(i3_LQ_LWC_c34585/f1p1/4026) and CYP92A6 (i1_LQ_LWC_c8195/f1p3/1783 and

i1_LQ_LWC_c36100/f1p0/1831) are all enriched and expressed in the radicles (Figure S4). In addition, D2 (i1_LQ_LWC_c2765/f2p1/2032) and CYP734A1 (i2_LQ_LWC_c108886/f1p0/2200), which inactivate BR through hydroxylation to maintain the steady state of $\mathrm{BR}$, also had higher expression levels in radicles (Figure S4). In summary, the genes that synthesize BR and maintain BR homeostasis in the radicles were very active, which ensured the normal level of $B R$. We speculated that this may explain why the elongation of radicles was significantly higher than that of the germs.

\section{Discussion}

Our results found that the time for different pearl millet seeds to reach the germination standard under the same environmental conditions is slightly different. But nearly all seeds reached the germination standard $24 \mathrm{~h}$ after the imbibition. Therefore, we think that pearl millet can be regarded as a representative plant with rapid seed germination. When pearl millet is planted in the field, it is not easy to be eroded by weeds, which may be related to the rapid growth of seedlings after the germination of seeds. In order to explore the growth rate of pearl millet seedlings, we measured the germ and radicle lengths of seedlings at $24 \mathrm{~h}, 36 \mathrm{~h}$, and $48 \mathrm{~h}$ after imbibition of pearl millet seeds. The result showed that there were extremely significant differences between each time point, and the average germ and radicle 
lengths reached $12.4 \mathrm{~mm}$ and $20.5 \mathrm{~mm}$ at $48 \mathrm{~h}$, respectively (Table S2). Compared with switchgrass, another energy model plant, its plant height reaches $20-30 \mathrm{~mm}$ in seedlings after 10 days of growth [11]. The growth rate of pearl millet seedlings is much faster than that of switchgrass. Therefore, pearl millet can also be regarded as a representative plant of rapid seedling growth.

In the previous studies, many researchers reported that GA can promote the seed germination [48-50]. It has been found in rice that the LOL1/bZIP58 complex can promote the synthesis of gibberellins and enhance the germination rate and speed of rice seeds [51]. The Arabidopsis GA deletion mutant ga 1 cannot germinate without exogenous GA $[13,52]$. In the signal transduction pathway of GA, the DELLA protein is the main suppressor, which binds to key downstream regulatory factors to inhibit the signal transduction of GA [31]. A research found that DELLA protein enhanced the degradation of PIF3, thereby inhibiting the elongation of Arabidopsis hypocotyl [32]. In addition to promoting seed germination, GA can also promote root growth by degrading DELLA protein [53]. In this study, we found that the GA signal transduction pathway was very active at $2 \mathrm{~h}$ after seeds imbibition, especially the genes such as GID1, PIF3 and PIF4 were highly expressed, while the inhibitory DELLA protein gene was not expressed, which is consistent with the results of previous studies. In addition, we measured the GA content of pearl millet seeds in the early stage after imbibition, and found that this hormone was accumulated in a large amount, and the content was much higher than that in switchgrass [14]. Considering the slow germination of switchgrass seeds, we speculated that the large amount of GA was accumulated in the early stage after seed imbibition is one of the reasons for the rapid germination of pearl millet seeds.

In addition, to promote plant leaf and root development, auxin also plays an active role in plant growth and development, such as regulating tissue differentiation, organogenesis and morphogenesis, apical dominance and flowering period [54-57]. Recent studies have shown that auxin stimulates the abscisic acid signals to induce the seed dormancy [58]. Therefore, the balance of auxin content is essential for seed germination and seedling development. In our study, we found that the GH3 gene, which promotes the degradation of auxin, was highly expressed in the auxin signal transduction pathway. The expression of this gene was induced by auxin and played a negative feedback regulation role. We speculated that this gene promotes the maintenance of auxin in a balanced state, which neither promotes seed dormancy nor hinders other aspects of plant growth and development.

CTK plays a broad role in plant growth. In addition, CTK promotes the cell division, inducing bud differentiation, removing apical advantages, and enhancing plant resistance. Many studies have also found that CTK efficiently promotes the seed development, increases the seed setting rate and breaks seed dormancy [59]. The earliest discovered and purified CTK was zeatin and isolated from immature corn seeds [60]. In the process of seed germination, GA cannot reach the point of action due to the barrier of cell compartments, but CTK can change the permeability of the membrane to promote GA to function [59]. In this study, we found that after $2 \mathrm{~h}$ of seed imbibition, the CTK signal transduction pathway was very active, and the receptor kinase CRE1 and class B response factor ARR-B genes were highly expressed. In addition, the biosynthetic pathway of zeatin was also significantly enriched at this stage (Table S5). Zeatin is the main active ingredient of CTK, and its biosynthesis is the guarantee for the 
function of CTK. Therefore, we speculated that the activation of zeatin biosynthesis pathway and the signal transduction pathway of CTK itself is one of the reasons for rapid germination of pearl millet seeds.

Previous studies reported that during the germination of tobacco and Arabidopsis seeds, the release of dormancy involves the light/gibberellin pathway and results in testa rupture $[8,61,62]$. In our study, in addition to the above-mentioned GA-related pathways, light-related pathways have also been detected. Photosynthesis plays the most important role in the growth and development of plants. As we know, light provides ATP and NADPH for the dark reaction stage of photosynthesis. In this stage, carbon dioxide is converted into carbohydrates to produce energy for plants. Ferredoxin, which acts as a regulator, can make the dark reaction work more effectively [63]. We checked the expression level of gene petF (i0_HQ_LWC_c109/f3p0/774, i0_LQ_LWC_c1036/f1p0/698 and i1_LQ_LWC_c26510/f1p0/1231), which encodes ferredoxin in the photosynthesis pathway, and found that it was very active in germs and got a peak at $36 \mathrm{~h}$ after seeds imbibition. At the same time, the photosystem subunits are highly expressed (Fig. 6), which all play an important role in photosynthesis. For example, the PSI-H subunit (i0_LQ_LWC_c641/f1p0/737) is necessary for the state transition in the light system, and this state change is a dynamic mechanism for plants to quickly respond to changes in light [64]. The PSI-D subunit in Arabidopsis is encoded by the PsaD gene (i0_HQ_LWC_c150/f3p0/679 and i0_LQ_LWC_c1898/f1p2/916). The PsaD mutant is seedling-lethal illustrate that they play an important role in maintaining the stability of the photosystem I [65]. In addition, studies have shown that photosystem II oxygen-promoting protein 2 (PsbP, i1_LQ_LWC_c42560/f1p4/1019 and i1_LQ_LWC_c38928/f1p0/1053) and photosystem II oxygen-promoting protein 3 (PsbQ, i0_LQ_LWC_c1522/f1p0/888) have specific and important roles in coordinating the activity of the donor and acceptor sides of PSII and stabilizing the active form of the PSII-light-harvesting complex II (LHCII) supercomplex [66]. In summary, we speculated that the formation of photosynthetic system at early seedlings stage provides sufficient energy for seedling development, which is a guarantee for the rapid growth of seedlings in pearl millet.

\section{Conclusion}

Germination experiments proved that pearl millet seeds germination and seedlings growth were very fast. Under suitable temperature and light conditions, the seeds can reach the germination standard at $24 \mathrm{~h}$ after seeds imbibition. In addition, seedlings experienced a rapid growth process during $24 \mathrm{~h}-36 \mathrm{~h}$ after seeds imbibition. Therefore, we regard pearl millet as a representative plant for rapid seed germination and seedling growth. Combined with transcriptomic methods, we characterized the gene expression of pearl millet seeds from germination to early seedling growth, and identified the key genes involved in this stage. WGCNA analysis showed that the functions of different gene clusters were highly organized and time specific, indicating that different genes were involved in the regulation of seed germination, germs and radicles growth. KEGG enrichment analysis showed that the differential expression of key genes in the GA, auxin and CTK signal transduction, photosynthesis, photosynthesis-antenna proteins, and brassinosteroids biosynthesis pathways regulated the growth of pearl millet seeds germination and 
growth of seedlings. In general, this study provided a reference method for solving problems for plants with slow seed germination and slow growth and development of seedlings. At the same time, it can be used as a transcriptome data resource for comparison and analysis when other plants are studying with the similar scientific problems.

\section{Methods}

\section{Plant materials}

The seeds of pearl millet cv. "Tifleaf 3" were used in our study and provided by key Laboratory Department of Grassland Science, Sichuan Agricultural University (Wenjiang, Sichuan, China). For the experiment, 200 seeds with uniform size and shape, and without damage were randomly selected. First,

30 seeds were randomly selected as the dry seed group (Seed), and the remaining 170 seeds were soaked in distilled water and shaken at $200 \mathrm{rpm}$ for 10 minutes to fully imbibe. Then 170 seeds were putted in a petri dish with a double-layer filter paper as a germination bed and all of them were maintained in an incubator at $30^{\circ} \mathrm{C} / 25^{\circ} \mathrm{C}$ (day/night) with a photoperiod of $16 \mathrm{~h} / 8 \mathrm{~h}$ (day/night). After 2 hours, 30 seeds were randomly selected as the $2 \mathrm{~h}$ after seed imbibition group (2HAIS). Further, seedlings have been formed after 24,36 and $48 \mathrm{~h}$ seeds imbibition. Thirty individual plants were randomly selected to measure the length of germs and radicles respectively. After the measurement, the germs and radicles were separated as the 24, 36 and $48 \mathrm{~h}$ germs groups (24HAIG, 36HAIG and $48 \mathrm{HIAG}$ ) and the radicles group (24HAIR, 36HAIR and 48HAIR). Finally, all the above materials were stored in liquid nitrogen for subsequent RNA extraction. The germination test of maize inbred line B73, rice cv. "Nipponbare", switchgrass cv. "Alamo" and orchardgrass cv. "Dianbei" seeds was carried out under the same culture conditions and the same naming rules as above were used. The germinated seeds were counted every $24 \mathrm{~h}$. The germination time of $50 \%$ of the seeds in the petri dish and the germination time of all the remaining seeds were recorded.

\section{RNA extraction and transcriptome sequencing}

Total RNA was extracted using the Direct-zol ${ }^{\mathrm{TM}}$ RNA MiniPrep Kit (Zymo Research Co.), following the manufacturer's instructions. After that, RNA purity, concentration and integrity were detected via the NanoPhotometer ${ }^{\circledR}$ spectrophotometer (IMPLEN, CA, USA), Qubit ${ }^{\circledR}$ RNA Assay Kit in Qubit ${ }^{\circledR} 2.0$ Flurometer (Life Technologies, CA, USA) and Nano 6000 Assay Kit and Agilent Bioanalyzer 2100 system (Agilent Technologies, CA, USA).

A total of $3 \mu \mathrm{g}$ RNA was used for the construction of cDNA library for each sample. Total 24 sequencing libraries were generated using NEBNext ${ }^{\circledR}$ Ultra ${ }^{\mathrm{TM}}$ RNA Library Prep Kit for Illumina ${ }^{\circledR}$ (NEB, USA) [67]. In order to ensure the quality of bio-analysis, raw reads containing adapters and low quality must be filtered to obtain clean reads for subsequent analysis. The filter criteria are as follows: 1) Remove reads with adapters. 2) Remove $\mathrm{N}$ ( $\mathrm{N}$ means that the base type cannot be determined) is greater than $10 \%$ of reads.

3) Remove low-quality reads (reads with $Q_{\text {phred }} \leq 20$ bases accounting for more than $50 \%$ of the entire 
read length). At the same time, the GC content of clean reads, and the values of Q20 and Q30 were also calculated.

\section{Quantification of gene expression level and identification of DEGs}

We use the Pacbio full-length transcriptome data of pearl millet as a reference sequence for analysis [6]. The classic transcriptome data processing includes two steps: sequence alignment and transcription abundance calculation. This process uses two software, TopHat2 and Cufflinks, but the combination of these two software needs high requirements on computer hardware and is very time-consuming. Here we used kallisto software for a new RNA sequence quantization method, which is close to the best in speed and accuracy $[68,69]$. The analysis method refers to the description in the article, briefly, using the cDNA data of the reference sequence to construct an index, and then to identify and quantify the transcript. The expression abundance of genes is expressed by transcripts per million (TPM) [70]. In order to ensure the accuracy and reliability of the experiment, we filtered out the genes with the maximum TPM less than 5 in the 24 samples. The differential gene identification software used with kallisto software is an $\mathrm{R}$ package called sleuth, which can quickly and accurately calculate the differentially expressed genes [71]. The genes (Q-value < 0.05) obtained by sleuth software were considered to be differentially expressed, and the significance of DEGs determined by the absolute value of log2 (Group1/Group2) $\geq 1$ as the threshold.

\section{WGCNA analysis and gene function annotation}

Due to the large number of sampling points in this study, we used R software to perform weighted gene co-expression network analysis (WGCNA) [72]. 24,307 genes with a TPM value greater than 5 in any sample and the absolute value of log2 (Group1/Group2) $\geq 1$ in at least one comparison group were used for WGCNA analysis, with a soft threshold power of 17. For functional annotations of genes, refer to the annotations in the pearl millet Pacbio full-length transcriptome [6]. For the genes in the modules, we use KOBAS software for KEGG enrichment analysis [73].

\section{GA content determination}

We used the same sampling method and strategy like RNA-seq to obtain samples to measure the GA content, the difference is that two time points 4 and $6 \mathrm{~h}$ after seed imbibition were added. Using the same naming rules, the samples at the two time points were named 4HAIS and 6HIAS. We used enzyme-linked immunosorbent assay to determine the content of GA [74]. The Plant Gibberellic Acid (GA) ELISA Kit is provided by Shanghai Enzyme-Linked Biology Company. The experimental method was carried out strictly in accordance with the instructions. Three biological replicates and three technical replicates were measured for each sample.

\section{Abbreviations}


KEGG : Kyoto Encyclopedia of Genes and Genomes; ABA: abscisic acid; GA: gibberellin; CTK: cytokinin; DLI: daily light integral; DEGs: differently expressed genes; WGCNA: weighted gene co-expression network analysis; NCBI: National Center for Biotechnology Information; IAA: indole acetic acid; LHC: lightharvesting complex; BR: brassinosteroid; cDNA: complementary DNA; RNA-Seq: RNA sequencing; TPM: transcripts per million

\section{Declarations}

\section{Acknowledgements}

The author would like to thank Novogene Bioinformatics Co., Ltd. (Beijing, China) for its assistance in sequencing and the BMK Cloud platform (www.biocloud.net) for completing part of the data analysis.

\section{Authors' contributions}

BW and LH designed research studies; BW and MS conducted experiments, acquired data, and analyzed data; $B W, D Y$, and $H Z$ wrote the manuscript; $X W, I K, Y Y, G N$ and GF revised the manuscript; $X Z, Z L$ and $Y P$ provided plant materials; All authors read and approved the final manuscript.

\section{Funding}

This work was supported by the Sichuan Province Research grant (2016NYZ0036), the Modern Agroindustry Technology Research System (CARS-34) and Modern Agricultural Industry System Sichuan Forage Innovation Team (SCCXTD-2020-16).

\section{Availability of data and materials}

The raw sequence data have been deposited in the NCBI database: PRJNA670183 (https://www.ncbi.nlm.nih.gov/sra/PRJNA670183).

\section{Ethics approval and consent to participate}

Not applicable.

\section{Competing interests}

The authors declare that they have no competing interests 


\section{Consent for publication}

Not applicable.

\section{References}

1. Koornneef M, Bentsink L, Hilhorst $\mathrm{H}$ : Seed dormancy and germination. Current opinion in plant biology 2002, 5(1):33-36.

2. Gallardo K, Job C, Groot SP, Puype M, Demol H, Vandekerckhove J, Job D: Importance of methionine biosynthesis for Arabidopsis seed germination and seedling growth. Physiologia plantarum 2002, 116(2):238-247.

3. Kim C, Lee T-H, Guo H, Chung SJ, Paterson AH, Kim D-S, Lee G-J: Sequencing of transcriptomes from two Miscanthus species reveals functional specificity in rhizomes, and clarifies evolutionary relationships. BMC plant biology 2014, 14(1):134.

4. Laougé ZB, Merdun H: Kinetic analysis of Pearl Millet (Penissetum glaucum (L.) R. Br.) under pyrolysis and combustion to investigate its bioenergy potential. Fuel 2020, 267:117172.

5. Patel B, Patel A, Gami B, Patel P: Energy balance, GHG emission and economy for cultivation of high biomass verities of bamboo, sorghum and pearl millet as energy crops at marginal ecologies of Gujarat state in India. Renewable Energy 2020, 148:816-823.

6. Sun M, Huang D, Zhang A, Khan I, Yan H, Wang X, Zhang X, Zhang J, Huang L: Transcriptome analysis of heat stress and drought stress in pearl millet based on Pacbio full-length transcriptome sequencing. BMC Plant Biology 2020, 20(1):1-15.

7. Singh R, Singh D, Tyagi P: Effect of Azotobacter, farmyard manure and nitrogen fertilization on productivity of pearl millet hybrids (Pennisetum glaucum (I) r. br) in semi-arid tropical environment. Archives of Agronomy and Soil Science 2003, 49(1):21-24.

8. Kucera B, Cohn MA, Leubner-Metzger G: Plant hormone interactions during seed dormancy release and germination. Seed Science Research 2005, 15(4):281-307.

9. Ross JJ, Weston DE, Davidson SE, Reid JB: Plant hormone interactions: how complex are they? Physiologia plantarum 2011, 141(4):299-309.

10. Santner A, Estelle M: Recent advances and emerging trends in plant hormone signalling. Nature 2009, 459(7250):1071-1078.

11. Karssen C, Brinkhorst-Van der Swan D, Breekland A, Koornneef M: Induction of dormancy during seed development by endogenous abscisic acid: studies on abscisic acid deficient genotypes of Arabidopsis thaliana (L.) Heynh. Planta 1983, 157(2):158-165.

12. Ali-Rachedi S, Bouinot D, Wagner M-H, Bonnet M, Sotta B, Grappin P, Jullien M: Changes in endogenous abscisic acid levels during dormancy release and maintenance of mature seeds: studies 
with the Cape Verde Islands ecotype, the dormant model of Arabidopsis thaliana. Planta 2004, 219(3):479-488.

13. Koornneef M, Van der Veen J: Induction and analysis of gibberellin sensitive mutants in Arabidopsis thaliana (L.) Heynh. Theoretical and Applied genetics 1980, 58(6):257-263.

14. Zhang S, Sun F, Wang W, Yang G, Zhang C, Wang Y, Liu S, Xi Y: Comparative transcriptome analysis provides key insights into seedling development in switchgrass (Panicum virgatum L.). Biotechnology for biofuels 2019, 12(1):193.

15. Qin G, Gu H, Zhao Y, Ma Z, Shi G, Yang Y, Pichersky E, Chen H, Liu M, Chen Z: An indole-3-acetic acid carboxyl methyltransferase regulates Arabidopsis leaf development. The Plant Cell 2005, 17(10):2693-2704.

16. Wang W, Xu B, Wang H, Li J, Huang H, Xu L: YUCCA genes are expressed in response to leaf adaxialabaxial juxtaposition and are required for leaf margin development. Plant Physiology 2011, 157(4):1805-1819.

17. Zhou W, Wei L, Xu J, Zhai Q, Jiang H, Chen R, Chen Q, Sun J, Chu J, Zhu L: Arabidopsis tyrosylprotein sulfotransferase acts in the auxin/PLETHORA pathway in regulating postembryonic maintenance of the root stem cell niche. The Plant Cell 2010, 22(11):3692-3709.

18. Liu H, Wang S, Yu X, Yu J, He X, Zhang S, Shou H, Wu P: ARL1, a LOB-domain protein required for adventitious root formation in rice. The Plant Journal 2005, 43(1):47-56.

19. Ren B, Liang Y, Deng Y, Chen Q, Zhang J, Yang X, Zuo J: Genome-wide comparative analysis of typeA Arabidopsis response regulator genes by overexpression studies reveals their diverse roles and regulatory mechanisms in cytokinin signaling. Cell research 2009, 19(10):1178-1190.

20. Zhao Y, Cheng S, Song Y, Huang Y, Zhou S, Liu X, Zhou D-X: The interaction between rice ERF3 and WOX11 promotes crown root development by regulating gene expression involved in cytokinin signaling. The Plant Cell 2015, 27(9):2469-2483.

21. Zou X, Shao J, Wang Q, Chen P, Zhu Y, Yin C: Supraoptimal cytokinin content inhibits rice seminal root growth by reducing root meristem size and cell length via increased ethylene content. International journal of molecular sciences 2018, 19(12):4051.

22. Sweet G, Wareing P: Role of plant growth in regulating photosynthesis. Nature 1966, 210(5031):7779.

23. Oh W, Cheon IH, Kim KS, Runkle ES: Photosynthetic daily light integral influences flowering time and crop characteristics of Cyclamen persicum. HortScience 2009, 44(2):341-344.

24. Garland KF, Burnett SE, Day ME, van lersel MW: Influence of substrate water content and daily light integral on photosynthesis, water use efficiency, and morphology of Heuchera americana. Journal of the American Society for Horticultural Science 2012, 137(1):57-67.

25. Pan X, Ma J, Su X, Cao P, Chang W, Liu Z, Zhang X, Li M: Structure of the maize photosystem I supercomplex with light-harvesting complexes I and II. Science 2018, 360(6393):1109-1113.

26. Stelpflug SC, Sekhon RS, Vaillancourt B, Hirsch CN, Buell CR, de Leon N, Kaeppler SM: An expanded maize gene expression atlas based on RNA sequencing and its use to explore root development. The 
plant genome 2016, 9(1):1-16.

27. Hsu S-K, Tung C-W: RNA-Seq analysis of diverse rice genotypes to identify the genes controlling coleoptile growth during submerged germination. Frontiers in plant science 2017, 8:762.

28. Wang S, Wang X, He Q, Liu X, Xu W, Li L, Gao J, Wang F: Transcriptome analysis of the roots at early and late seedling stages using Illumina paired-end sequencing and development of EST-SSR markers in radish. Plant cell reports 2012, 31(8):1437-1447.

29. Chen X, Yang H, Gan C, Yuan R, Han Z, Li Y: Transcriptomic analysis of the phytotoxic effects of 1allyl-3-methylimidazolium chloride on the growth and plant hormone metabolic pathways of maize (Zea mays L.) seedlings. Chemosphere 2020, 241:125013.

30. Liu W-Y, Chang Y-M, Chen SC-C, Lu C-H, Wu Y-H, Lu M-YJ, Chen D-R, Shih AC-C, Sheue C-R, Huang HC: Anatomical and transcriptional dynamics of maize embryonic leaves during seed germination. Proceedings of the National Academy of Sciences 2013, 110(10):3979-3984.

31. Liu X, Hu P, Huang M, Tang Y, Li Y, Li L, Hou X: The NF-YC-RGL2 module integrates GA and ABA signalling to regulate seed germination in Arabidopsis. Nature Communications 2016, 7(1):1-14.

32. Li K, Yu R, Fan L-M, Wei N, Chen H, Deng XW: DELLA-mediated PIF degradation contributes to coordination of light and gibberellin signalling in Arabidopsis. Nature communications 2016, 7(1):111.

33. Ou Y, Lu X, Zi Q, Xun Q, Zhang J, Wu Y, Shi H, Wei Z, Zhao B, Zhang X: RGF1 INSENSITIVE 1 to 5, a group of LRR receptor-like kinases, are essential for the perception of root meristem growth factor 1 in Arabidopsis thaliana. Cell research 2016, 26(6):686-698.

34. Qi J, Wang Y, Yu T, Cunha A, Wu B, Vernoux T, Meyerowitz E, Jiao Y: Auxin depletion from leaf primordia contributes to organ patterning. Proceedings of the National Academy of Sciences 2014, 111(52):18769-18774.

35. Mellor N, Bennett MJ, King JR: GH3-mediated auxin conjugation can result in either transient or oscillatory transcriptional auxin responses. Bulletin of mathematical biology 2016, 78(2):210-234.

36. Stamm P, Kumar PP: Auxin and gibberellin responsive Arabidopsis SMALL AUXIN UP RNA36 regulates hypocotyl elongation in the light. Plant cell reports 2013, 32(6):759-769.

37. Spartz AK, Lee SH, Wenger JP, Gonzalez N, Itoh H, Inzé D, Peer WA, Murphy AS, Overvoorde PJ, Gray WM: The SAUR19 subfamily of SMALL AUXIN UP RNA genes promote cell expansion. The Plant Journal 2012, 70(6):978-990.

38. Yamburenko MV, Kieber JJ, Schaller GE: Dynamic patterns of expression for genes regulating cytokinin metabolism and signaling during rice inflorescence development. PloS one 2017, 12(4): $\mathrm{e} 0176060$.

39. Waldie T, Leyser O: Cytokinin targets auxin transport to promote shoot branching. Plant physiology 2018, 177(2):803-818.

40. Yamada H, Suzuki T, Terada K, Takei K, Ishikawa K, Miwa K, Yamashino T, Mizuno T: The Arabidopsis AHK4 histidine kinase is a cytokinin-binding receptor that transduces cytokinin signals across the membrane. Plant and Cell Physiology 2001, 42(9):1017-1023. 
41. Nelson N, Yocum CF: Structure and function of photosystems I and II. Annu Rev Plant Biol 2006, 57:521-565.

42. Dekker JP, Boekema EJ: Supramolecular organization of thylakoid membrane proteins in green plants. Biochimica et Biophysica Acta (BBA)-Bioenergetics 2005, 1706(1-2):12-39.

43. Drop B, Webber-Birungi M, Yadav SK, Filipowicz-Szymanska A, Fusetti F, Boekema EJ, Croce R: Lightharvesting complex II (LHCII) and its supramolecular organization in Chlamydomonas reinhardtii. Biochimica et Biophysica Acta (BBA)-Bioenergetics 2014, 1837(1):63-72.

44. Standfuss J, Terwisscha van Scheltinga AC, Lamborghini M, Kühlbrandt W: Mechanisms of photoprotection and nonphotochemical quenching in pea light-harvesting complex at $2.5 \AA$ resolution. The EMBO journal 2005, 24(5):919-928.

45. Clouse SD, Langford M, McMorris TC: A brassinosteroid-insensitive mutant in Arabidopsis thaliana exhibits multiple defects in growth and development. Plant physiology 1996, 111(3):671-678.

46. Wang L, Xu Y-Y, Ma Q-B, Li D, Xu Z-H, Chong K: Heterotrimeric G protein a subunit is involved in rice brassinosteroid response. Cell research 2006, 16(12):916-922.

47. Mouchel CF, Osmont KS, Hardtke CS: BRX mediates feedback between brassinosteroid levels and auxin signalling in root growth. Nature 2006, 443(7110):458-461.

48. Spicer PB, Dionne LA: Use of gibberellin to hasten germination of Solanum seed. Nature 1961, 189(4761):327-328.

49. Sun T-p: Gibberellin metabolism, perception and signaling pathways in Arabidopsis. The Arabidopsis Book/American Society of Plant Biologists 2008, 6.

50. Ogawa M, Hanada A, Yamauchi Y, Kuwahara A, Kamiya Y, Yamaguchi S: Gibberellin biosynthesis and response during Arabidopsis seed germination. The Plant Cell 2003, 15(7):1591-1604.

51. Wu J, Zhu C, Pang J, Zhang X, Yang C, Xia G, Tian Y, He C: Os LOL 1, a C 2 C 2-type zinc finger protein, interacts with 0 sb ZIP 58 to promote seed germination through the modulation of gibberellin biosynthesis in Oryza sativa. The Plant Journal 2014, 80(6):1118-1130.

52. Sun T, Kamiya Y: The Arabidopsis GA1 locus encodes the cyclase ent-kaurene synthetase A of gibberellin biosynthesis. The Plant Cell 1994, 6(10):1509-1518.

53. Tan H, Man C, Xie Y, Yan J, Chu J, Huang J: A crucial role of GA-regulated flavonol biosynthesis in root growth of Arabidopsis. Molecular plant 2019, 12(4):521-537.

54. Reinhardt D: Vascular patterning: more than just auxin? Current Biology 2003, 13(12):R485-R487.

55. Bohn-Courseau I: Auxin: a major regulator of organogenesis. Comptes rendus biologies 2010, 333(4):290-296.

56. Kepinski S, Leyser O: Plant development: auxin in loops. Current Biology 2005, 15(6):R208-R210.

57. van Doorn WG, Dole I, Çelikel FG, Harkema H: Opening of Iris flowers is regulated by endogenous auxins. Journal of Plant Physiology 2013, 170(2):161-164.

58. Liu X, Zhang H, Zhao Y, Feng Z, Li Q, Yang H-Q, Luan S, Li J, He Z-H: Auxin controls seed dormancy through stimulation of abscisic acid signaling by inducing ARF-mediated ABI3 activation in 
Arabidopsis. Proceedings of the National Academy of Sciences 2013, 110(38):15485-15490.

59. Thomas T: Cytokinins, cytokinin active compounds and seed germination. Physiology and Biochemistry of Seed Dormancy and Germination 1977.

60. Letham D, Shannon J, McDonald I: Regulators of cell division in plant tissues-III: The identity of Zeatin. Tetrahedron 1967, 23(1):479-486.

61. Leubner-Metzger G: Functions and regulation of [beta]-1, 3-glucanases during seed germination, dormancy release and after-ripening. Seed Science Research 2003, 13(1):17.

62. Liu PP, Koizuka N, Homrichhausen TM, Hewitt JR, Martin RC, Nonogaki H: Large-scale screening of Arabidopsis enhancer-trap lines for seed germination-associated genes. The Plant Journal 2005, 41(6):936-944.

63. Buchanan BB: Regulation of $\mathrm{CO} 2$ assimilation in oxygenic photosynthesis: the ferredoxin/thioredoxin system: perspective on its discovery, present status, and future development. Archives of biochemistry and biophysics 1991, 288(1):1-9.

64. Lunde C, Jensen PE, Haldrup A, Knoetzel J, Scheller HV: The PSI-H subunit of photosystem I is essential for state transitions in plant photosynthesis. Nature 2000, 408(6812):613-615.

65. Ihnatowicz A, Pesaresi P, Varotto C, Richly E, Schneider A, Jahns P, Salamini F, Leister D: Mutants for photosystem I subunit D of Arabidopsis thaliana: effects on photosynthesis, photosystem I stability and expression of nuclear genes for chloroplast functions. The Plant Journal 2004, 37(6):839-852.

66. Ifuku K, Ido K, Sato F: Molecular functions of PsbP and PsbQ proteins in the photosystem II supercomplex. Journal of Photochemistry and Photobiology B: Biology 2011, 104(1-2):158-164.

67. Wang Z, Gerstein M, Snyder M: RNA-Seq: a revolutionary tool for transcriptomics. Nature reviews genetics 2009, 10(1):57-63.

68. Bray NL, Pimentel H, Melsted P, Pachter L: Near-optimal probabilistic RNA-seq quantification. Nature biotechnology 2016, 34(5):525-527.

69. Conesa A, Madrigal P, Tarazona S, Gomez-Cabrero D, Cervera A, McPherson A, Szcześniak MW, Gaffney DJ, Elo LL, Zhang X: A survey of best practices for RNA-seq data analysis. Genome biology 2016, 17(1):13.

70. Vera Alvarez R, Pongor LS, Mariño-Ramírez L, Landsman D: TPMCalculator: one-step software to quantify mRNA abundance of genomic features. Bioinformatics 2019, 35(11):1960-1962.

71. Pimentel H, Bray NL, Puente S, Melsted P, Pachter L: Differential analysis of RNA-seq incorporating quantification uncertainty. Nature methods 2017, 14(7):687.

72. Langfelder P, Horvath S: WGCNA: an R package for weighted correlation network analysis. BMC bioinformatics 2008, 9(1):559.

73. Mao X, Cai T, Olyarchuk JG, Wei L: Automated genome annotation and pathway identification using the KEGG Orthology (KO) as a controlled vocabulary. Bioinformatics 2005, 21(19):3787-3793.

74. Inomata N, Miyakawa M, Ikeda N, Oda K, Aihara M: Identification of gibberellin-regulated protein as a new allergen in orange allergy. Clinical \& Experimental Allergy 2018, 48(11):1509-1520. 
Figures

$\mathbf{a}$

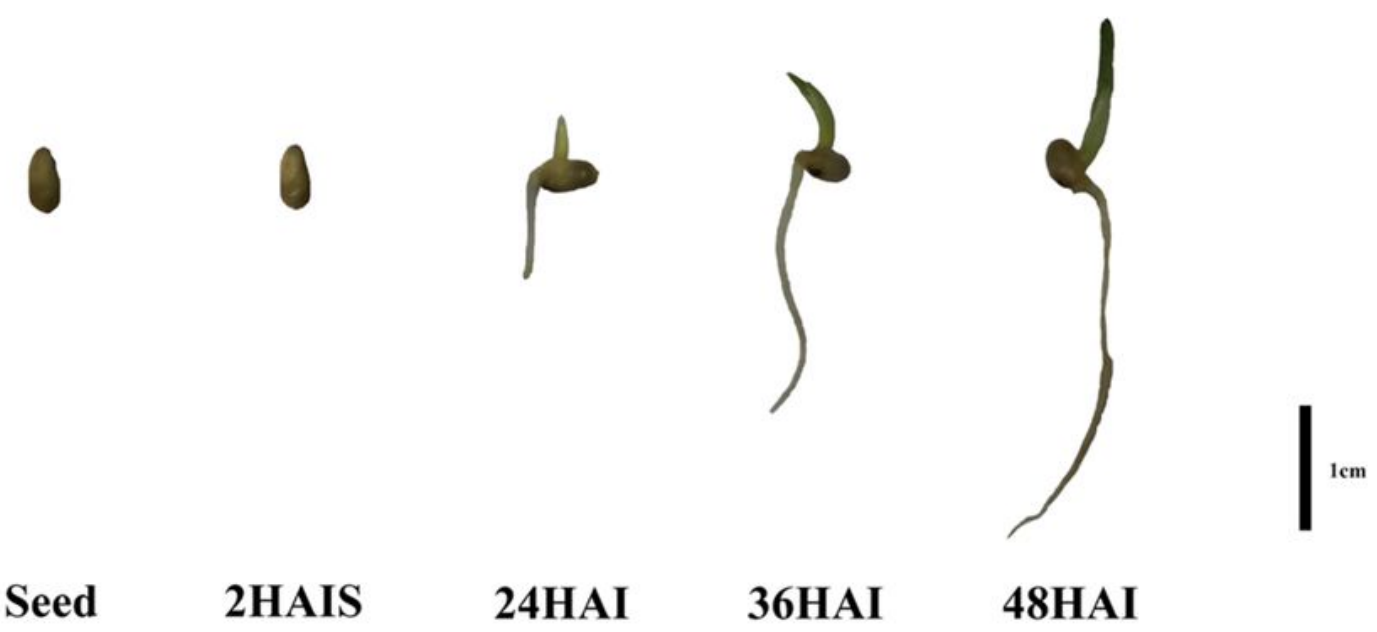

b

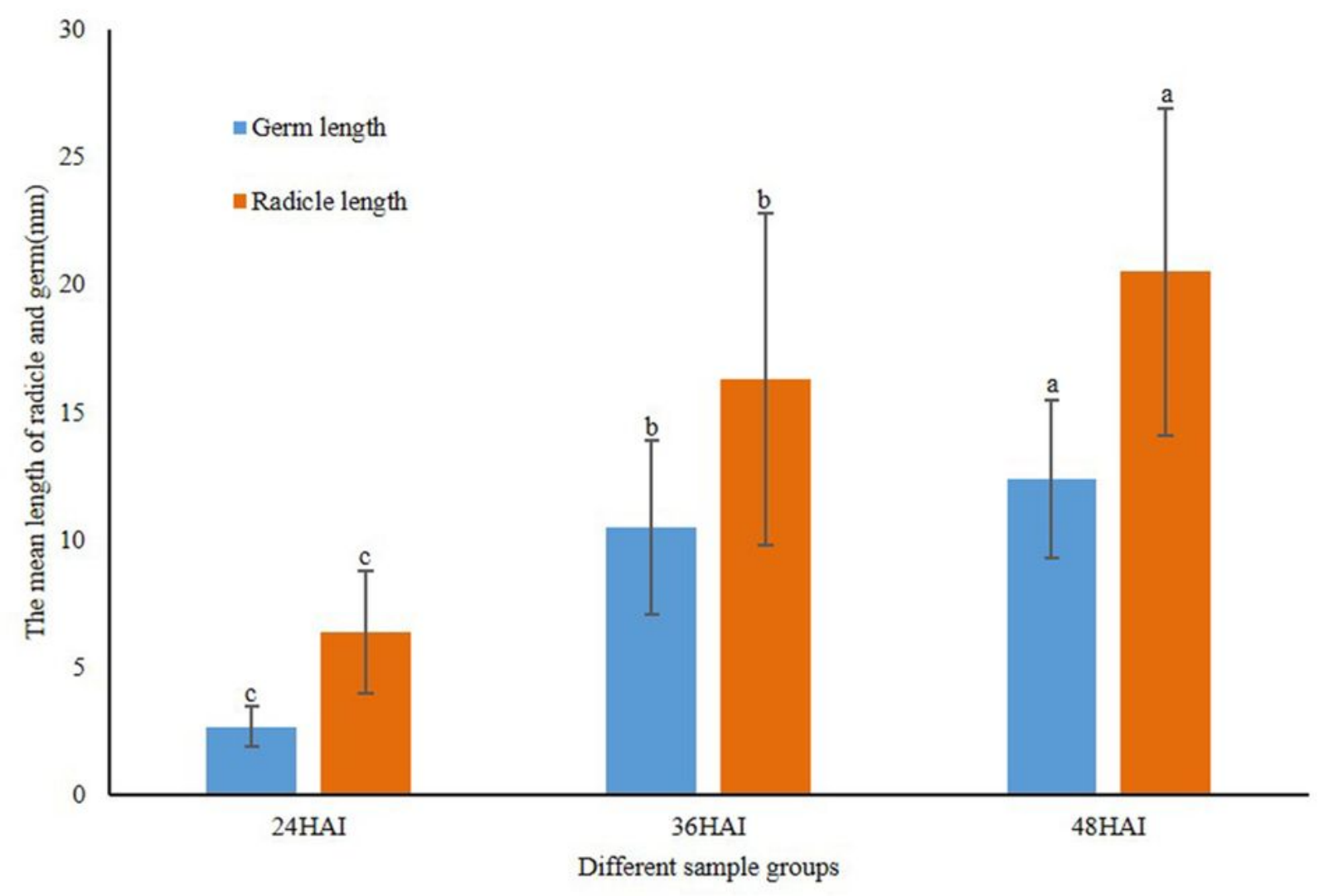

Figure 1

Changes of pearl millet seeds after imbibition. a. Morphological changes of pearl millet seeds from dry seed to seedling. $b$. The lengths of germ and radicle at different time points. Different lowercase letters indicate significant difference at $P<0.05$ 
$\mathbf{a}$

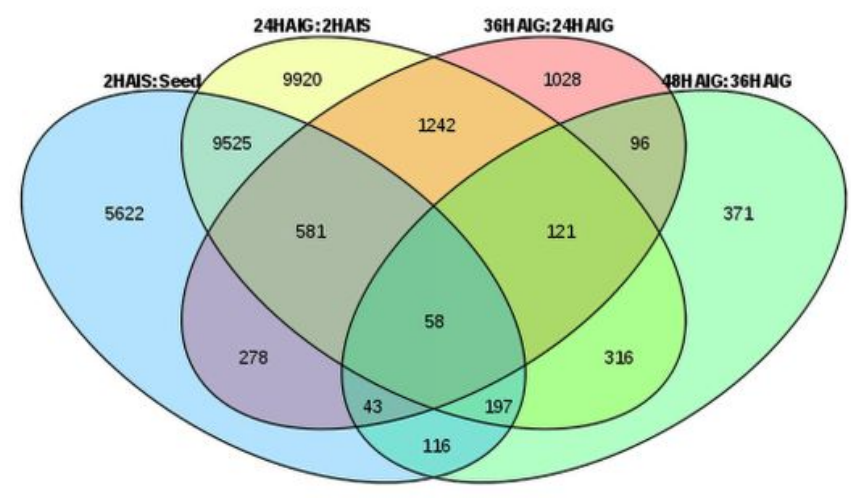

b

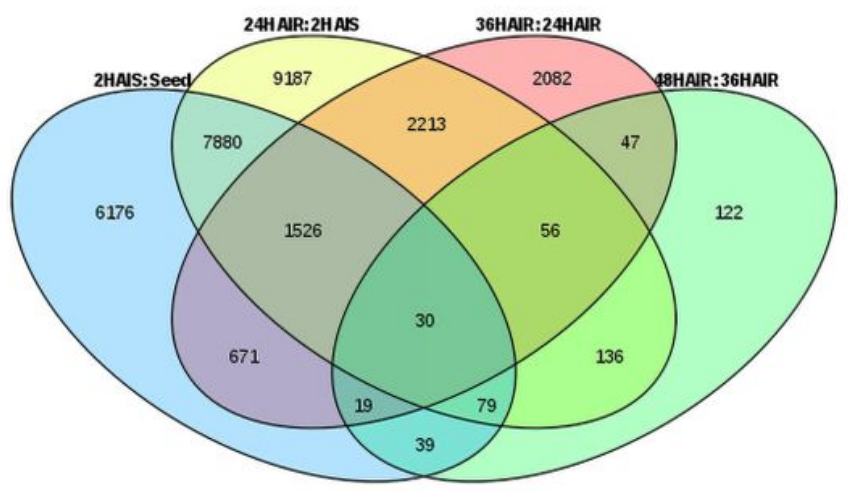

\section{Figure 2}

Overlap of differentially expressed genes in different comparison groups. a. Germ. b. Radicle 2

\section{Figure 3}

Weighted gene co-expression network analysis (WGCNA) results of DEGs. a. Cluster dendrogram. b. Module-trait relationships. c. Expression heat map and expression level of genes in the module. d. KEGG enrichment of genes in the module 


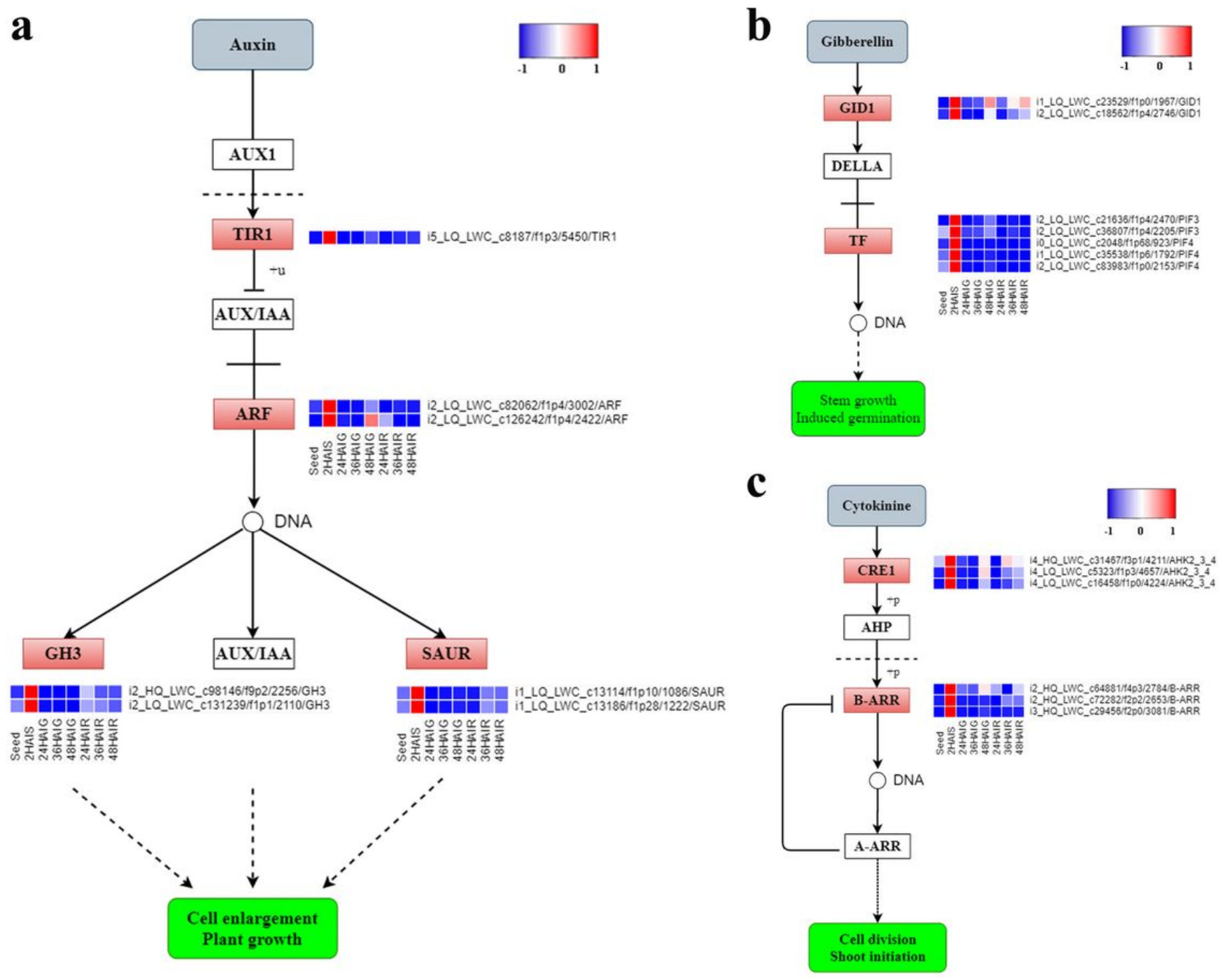

\section{Figure 4}

The expression pattern of key genes in hormone signal transduction pathway. a. The pathway in auxin signal transduction. b. The pathway in Gibberellin signal transduction. c. The pathway in Cytokinin signal transduction. The red rectangle indicates that the gene is enriched in the pathway. The expression data are the TPM values of the samples, red color indicates upregulated expression, and blue indicates downregulated expression 


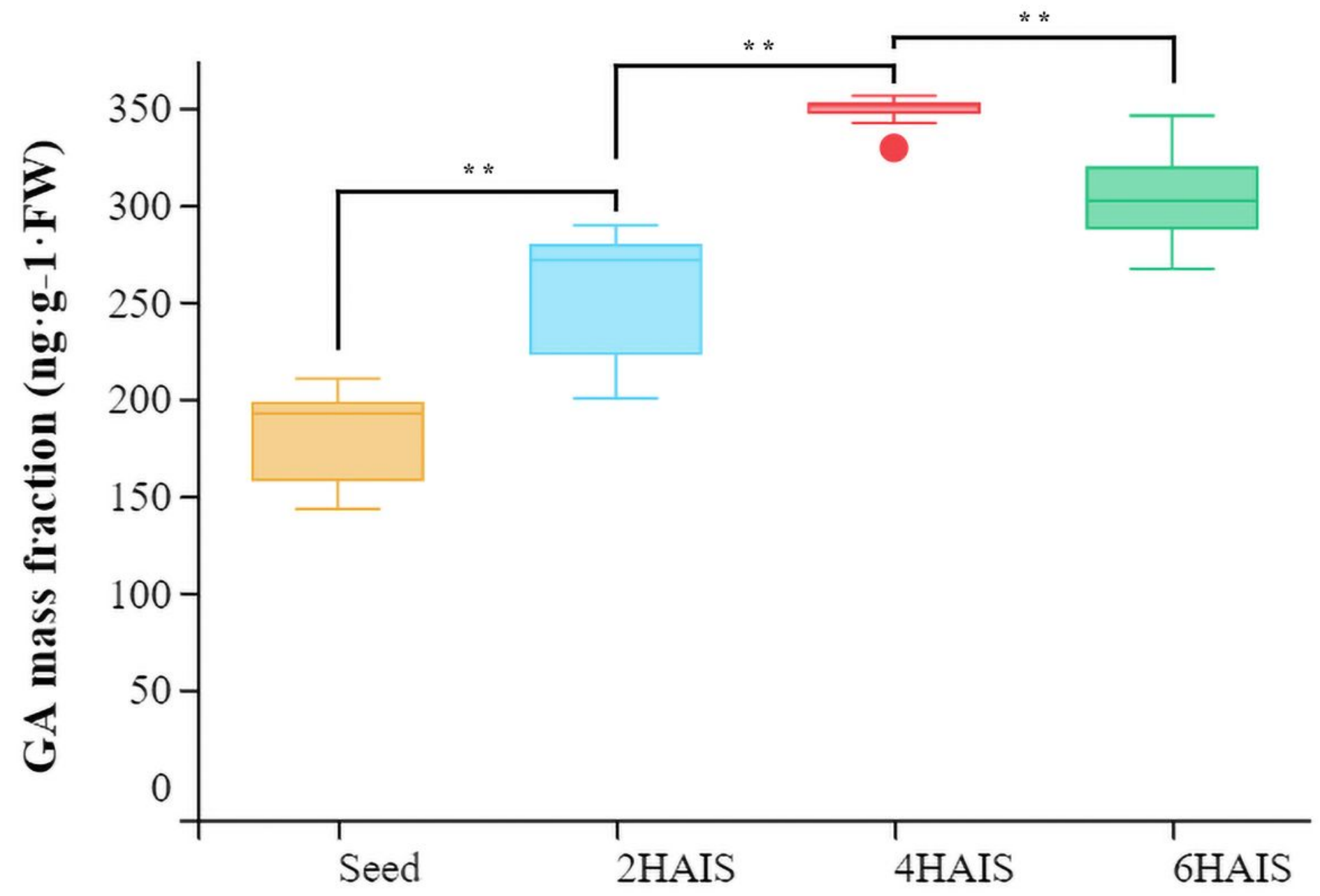

Figure 5

Gibberellin content at different time points. ** indicates significant difference at $\mathrm{P}<0.01$ 
a

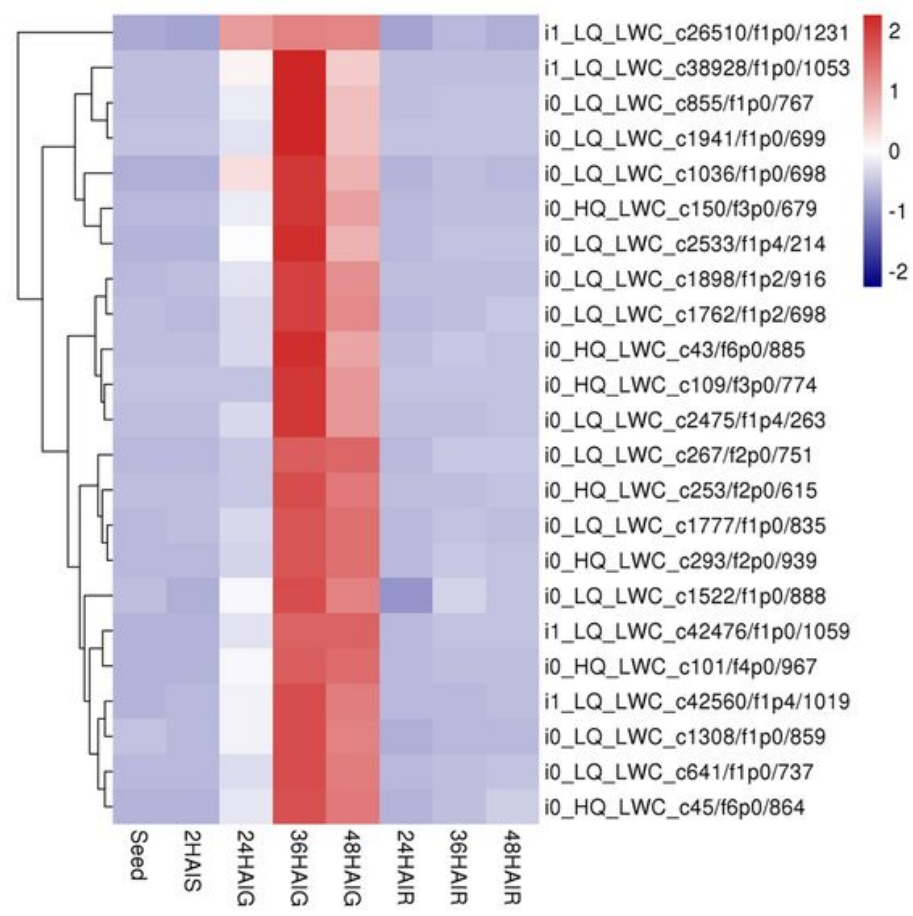

b

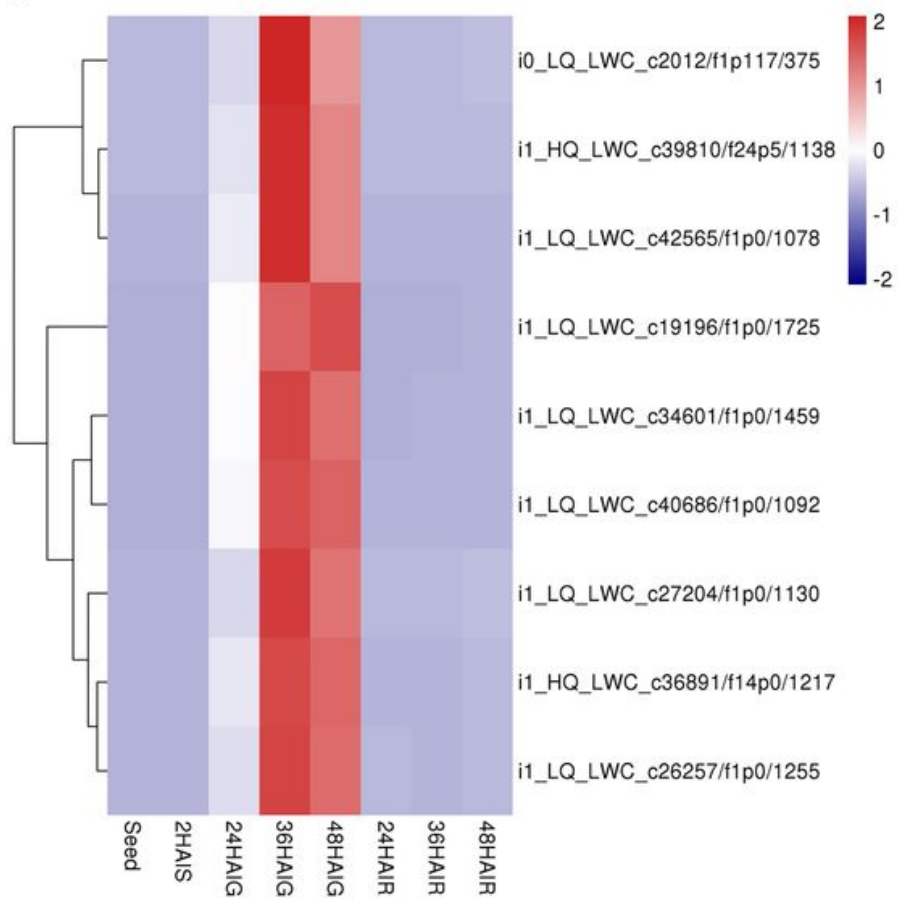

Figure 6

Heat map of genes expression related to light pathway. a. The genes in the photosynthetic pathway. b. The genes in the photosynthesis-antenna proteins pathway. The expression data are the TPM values of the samples, red color indicates upregulated expression, and blue indicates downregulated expression

\section{Supplementary Files}

This is a list of supplementary files associated with this preprint. Click to download.

- FigS1.jpg

- FigS2.jpg

- FigS3.jpg

- FigS4.jpg

- SupplementTables.xlsx 